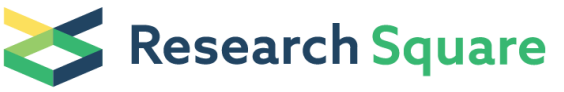

\section{Quantum-limit-breaking Magnetic Gradiometer with Entangled Light}

\section{Shuhe Wu}

Shanghai Jiao Tong University

Guzhi Bao ( $\nabla$ guzhibao@sjtu.edu.cn )

Shanghai Jiao Tong University

Jinxian Guo

Shanghai Jiao Tong University

Jun Chen

Shanghai Jiao Tong University

Wei Du

Shanghai Jiao Tong University

\section{Peiyu Yang}

Shanghai Jiao Tong University

\section{Chen}

Department of Physics and Electronic Science, East China Normal University, Shanghai 200062

\section{Weiping Zhang}

Shanghai Jiao Tong University

\section{Article}

Keywords:

Posted Date: February 10th, 2022

DOI: https://doi.org/10.21203/rs.3.rs-1249344/v1

License: (c) (1) This work is licensed under a Creative Commons Attribution 4.0 International License.

Read Full License

Additional Declarations: There is NO Competing Interest. 


\title{
Quantum-limit-breaking Magnetic Gradiometer with Entangled Light
}

\author{
Shuhe $\mathrm{Wu}^{1}$, Guzhi Bao ${ }^{1}{ }^{*}{ }^{\text {Jinxian Guo }}{ }^{1}$, Jun Chen ${ }^{1}$, Wei \\ $\mathrm{Du}^{1}$, Peiyu Yang ${ }^{1}$, L. Q. Chen ${ }^{2},{ }^{\dagger}$ and Weiping Zhang ${ }^{1,3,4 \ddagger}$ \\ 1 School of Physics and Astronomy, and Tsung-Dao Lee institute, \\ Shanghai Jiao Tong University, Shanghai 200240, P.R.China. \\ 2 State Key Laboratory of Precision Spectroscopy, \\ Quantum Institute for Light and Atom, Department of Physics, \\ East China Normal University, Shanghai 200062, P. R. China. \\ 3 Collaborative Innovation Center of Extreme Optics, \\ Shanxi University, Taiyuan, Shanxi 030006, China. \\ 4 Shanghai Research Center for Quantum Sciences, Shanghai 201315, China.
}

(Dated: January 11, 2022)

In the last few decades, the atomic magnetometry [1-7] has experienced remarkable development and reached to an outstanding sensitivity. This exciting technology is finding applications in various of fields [8-18]. The last two obstacles to further improve the sensitivity of magnetometry are quantum fluctuations and ambient magnetic field. For magnetometry based on optical readout of atomic ensemble's spin precession, the fundamental limitation of sensitivity is restricted by spin projection noise (SPN) and photon shot noise (PSN). Meanwhile, in practical applications, ambient magnetic field noise which is regarded as a common-mode magneticfield (CMM) noise is also limiting the sensitivity. Several techniques for reducing PSN [19-25], SPN [26, 27], and CMM noise [7, 28-31] have been proposed, respectively. To achieve the best sensitivity, it is essential to find an efficacious way to eliminate the noises from different sources, simultaneously. For this purpose, here we demonstrate a sub-shot-noise magnetic gradiometer utilizing entangled optical detection with quantumcorrelated twin beams. This leads to the simultaneous suppression of PSN and CMM noise. The quantum enhancement spans a frequency range from $7 \mathrm{~Hz}$ to $6 \mathrm{MHz}$ with maximum squeezing of $5.5 \mathrm{~dB}$ below the standard quantum limit (SQL). The sensitivity of gradient magnetic field reaches $18 \mathrm{fT} / \mathrm{cm} \sqrt{\mathrm{Hz}}$ at $20 \mathrm{~Hz}$ with these sophisticated techniques. Our study opens up new possibilities to use quantum-enhanced technology in developing sensitive magnetometry for practical applications with noisy and physically demanding environments.

Precision measurement technology based on atomic magnetometry with high sensitivity $[1-7]$ has great potential for applications in various fields, such as fundamental physics [8-11], biomag [12-14], materials science

\footnotetext{
*guzhibao@sjtu.edu.cn

$\dagger$ lqchen@phy.ecnu.edu.cn

$\ddagger$ wpz@sjtu.edu.cn
}

[15-17] and geology [18]. A practical magnetometer for applications requires a fT-level sensitivity at the frequencies below $\mathrm{kHz}$, which is limited by the ambient magnetic field noise and quantum noise. The SQL-limited sensitivity of atomic magnetometers has been greatly improved with numerous number of photons $N_{p}$ by increasing the power of the optical field and atoms $N_{a}$ by increasing the operation temperature of atomic vapor [1-7]. Operation of such a sensitive magnetometer is challenged by the ambient magnetic field noise, especially in an unshielded environment as shown in Fig. 1 (a). In order to eliminate the influence of noisy environment and achieve a highly effective cancellation of the CMM noise, the usual way is to operate a pair of magnetometers, one of which serves as a reference sensor in a gradiometric configuration [7, 28-31] as shown in Fig. 1 (b). The utilization of the reference sensor is to monitor the ambient magnetic field noise, but at the price of increasing quantum fluctuations due to the totally doubled number of particles in the configuration. To further improve the sensitivity in such a situation, it is essential to exploit quantum technologies to squeeze the quantum noise below the PSN [19-25] or SPN [26, 27].

Here, we propose and demonstrate a quantum gradiometer reducing both CMM noise and PSN at frequencies of $1-100 \mathrm{~Hz}$ by combining entangled twin beams and gradiometric detection into nonlinear magnetic-optical rotation (NMOR) magnetometry. Compared with reported quantum magnetometers which only squeeze the quantum noise in a single sensor, this work employs the entangled-light detection to achieve quantum enhancement in the differential measurement protocol [32-36] as shown in Fig. 1 (c). By implementing entangled twin beams in two fully polarized atomic ensembles as probe beams to couple the magnetic field into the polarization of the beams, the PSN can be broken by quantum entanglement, and meanwhile the CMM noise can be removed by gradiometric detection as well. As a result, we reach a gradient field sensitivity of $18 \mathrm{fT} / \mathrm{cm} \sqrt{\mathrm{Hz}}$ below the PSN by $5.5 \mathrm{~dB}$ at $20 \mathrm{~Hz}$ in a noisy environment. Our scheme shows the advantages of utilizing quantum technology in the presence of ambient magnetic noise to bring quantum-enhanced magnetometry into the realm of practical applications. 

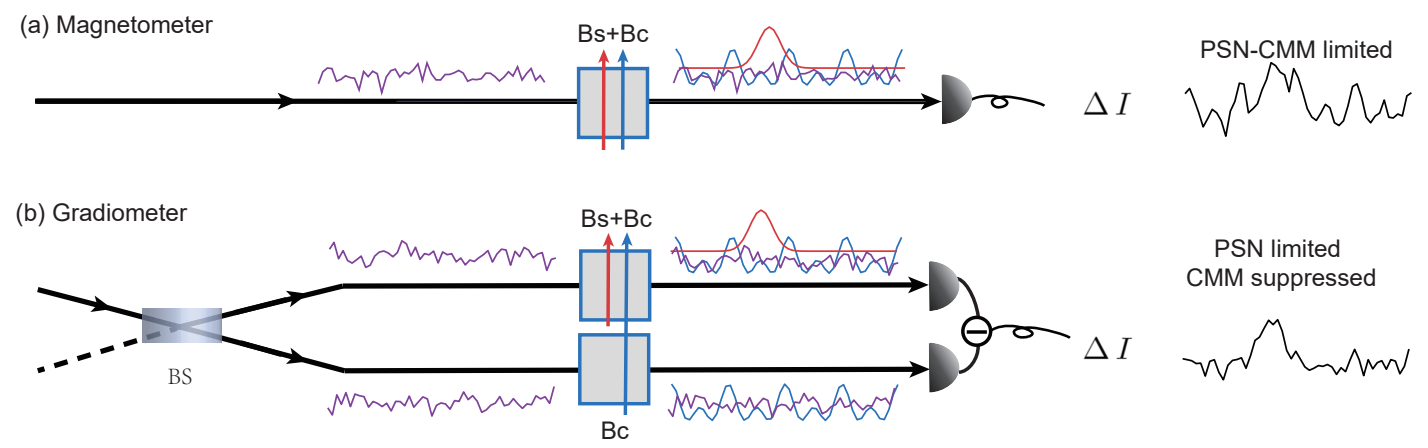

(c) Quantum- entangled gradiometer

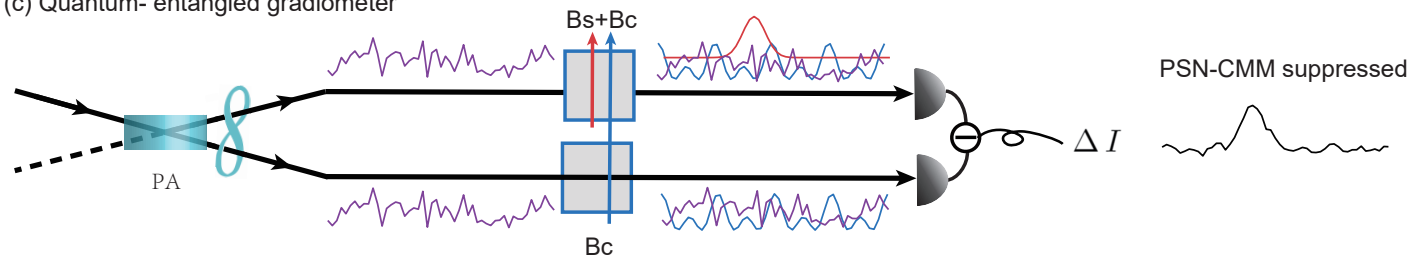

(d)

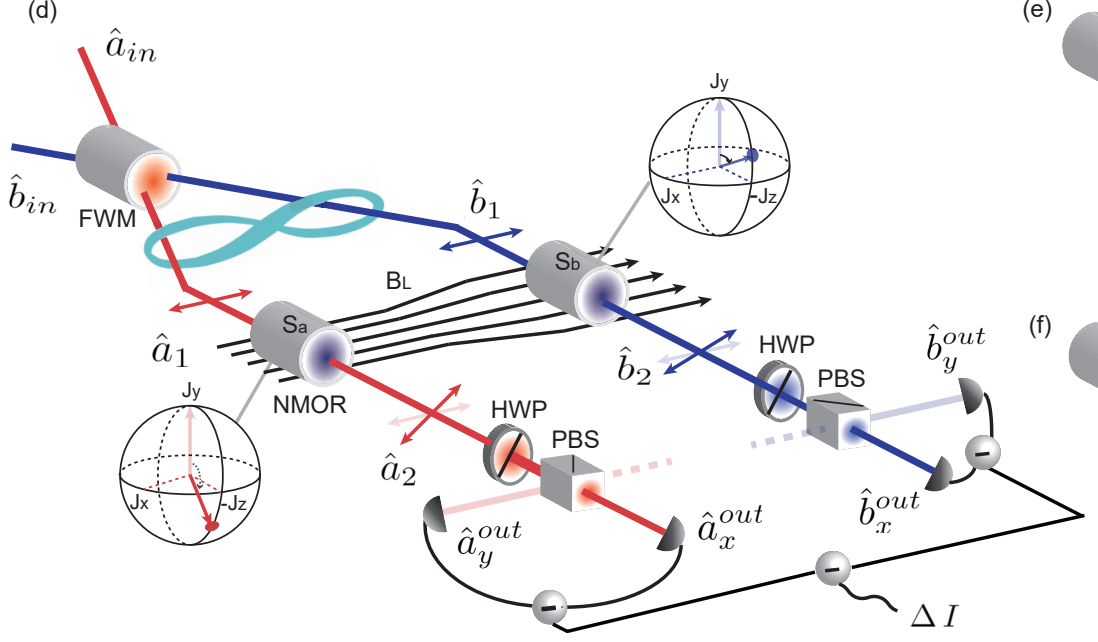

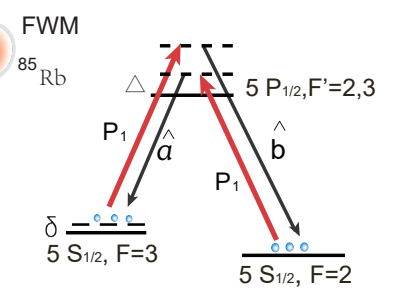

NMOR

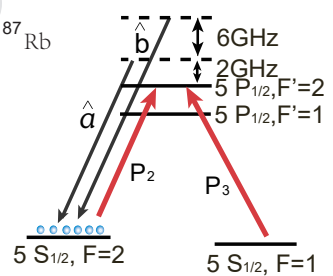

FIG. 1. (a,b,c) The protocols of magnetometer. In the case of single sensor (a), the noise of magnetometer is limited by both PSN and CMM noise. For the coherent gradiometer (b), the measured CMM noise of two sensor are identical while the quantum noise are uncorrelated. With differential measurement, the CMM noise is suppressed while the PSN is increased due to the doubled photon number. When the entangled twin beams are used as the probe fields for the magnetic measurement (c), both quantum noises and magnetic noises of two sensors are correlated. The quantum noise and magnetic field noise are effectively reduced, simultaneously. BS: 50/50 beam splitter. PA: parametric amplifier. PBS: polarizing beam splitter. $B_{c}$ : CMM noise. $B_{s}$ : magnetic signal. (d) Schematic diagram of the entangled gradiometer. HWP: half-wave plate. $B_{L}$ : leading magnetic field. $(\mathrm{e}, \mathrm{f})$ Energy level diagram in the $D_{1}$ line of ${ }^{85} \mathrm{Rb}$ for four-wave-mixing (FWM) process and energy level diagram in the $D_{1}$ line of ${ }^{87} \mathrm{Rb}$ for NMOR. $\Delta$ : one-photon detuning; $\delta$ : two-photon detuning. $\mathrm{P}_{1}$ : FWM pump field. $\mathrm{P}_{2}$ : optical pumping of NMOR. $\mathrm{P}_{3}$ : optical repumping of NMOR.

\section{Results.}

The quantum gradiometer consists of two parts: generation of entangled twin beams and a magnetic gradiometer. The schematic diagram is shown in Fig. 1 (d). Entangled twin beams $\hat{a}_{1}$ and $\hat{b}_{1}$ act as the probe beams of two atomic sensors to couple magnetic field $\mathrm{B}$ into their polarizations via NMOR as $\hat{a}_{2}$ and $\hat{b}_{2}$. $\hat{a}_{2}$ and $\hat{b}_{2}$ are split at the polarization beam splitter (PBS).
The output fields, designated $\hat{a}_{x}^{\text {out }}, \hat{a}_{y}^{\text {out }}, \hat{b}_{x}^{\text {out }}$ and $\hat{b}_{y}^{\text {out }}$, are detected to achieve optical rotation signals. The experimental setup is shown in Fig. 2, and the details are mentioned in the Methods. Below, we will give the experimental operation and results. We focus on how to reduce quantum noise and eliminate CMM noise at frequencies of $1-100 \mathrm{~Hz}$ in experimental operation.

Twin beams. Entangled twin beams are generated by a FWM process in ${ }^{85} \mathrm{Rb}$ atomic vapor $[32-36]$. A horizontally-polarized pump laser of $100 \mathrm{~mW}$ and an 


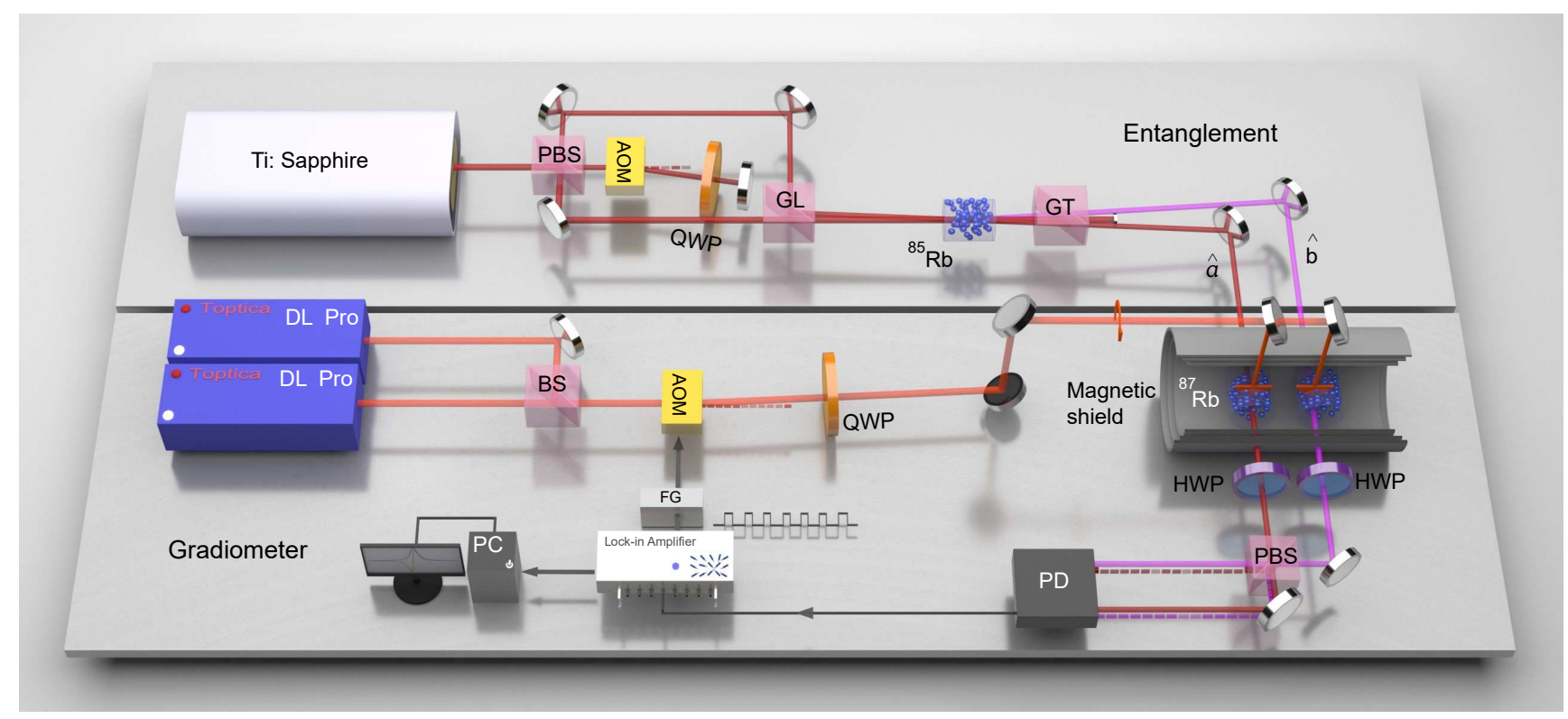

FIG. 2. Experimental setup. AOM: acousto-optic modulator. BS: 50/50 beam splitter. HWP: half-wave plate. PBS: polarizing beam splitter. QWP: quarter-wave plate. FG: function generator. GL: Glan-laser polarizer. GT: Glan-Thomson polarizer. PD: Photo-detector.

orthogonality-polarized signal field $a_{i n}$ of $10 \mu \mathrm{W}$ are combined by a Glan-Laser polarizer and sent into an ${ }^{85} \mathrm{Rb}$ vapor cell. The generated twin beams, designated $\hat{a}_{1}$ and $\hat{b}_{1}$, can be given as

$$
\begin{aligned}
& \hat{a}_{1}=G \hat{a}_{i n}+g \hat{b}_{i n}^{\dagger}, \\
& \hat{b}_{1}=G \hat{b}_{i n}+g \hat{a}_{i n}^{\dagger},
\end{aligned}
$$

where $G$ and $g$ are the gain factors satisfying $G^{2}-g^{2}=1$. $\hat{a}_{i n}$ and $\hat{b}_{i n}$ are two input fields for the FWM process. The frequency of the pump field is $1 \mathrm{GHz}$ blue detuned from the transition $\left|5 S_{1 / 2}, F=2\right\rangle \rightarrow\left|5 P_{1 / 2}, F^{\prime}=2\right\rangle$ of ${ }^{85} \mathrm{Rb}$ atom. After the ${ }^{85} \mathrm{Rb}$ cell, a Glan- Thomson (GT) polarizer is used to filter out the pump beam. The generated twin beams $\hat{a}_{1}$ and $\hat{b}_{1}$ are both $\sim 150 \mu \mathrm{W}$ in power, with $\mathrm{G}=3.9$ and horizontally polarized along the $\hat{x}$ direction. The squeezing spans a frequency range of $6 \mathrm{MHz}$. The maximum squeezing is $7 \mathrm{~dB}$ at $0.6 \mathrm{MHz}$.

Quantum gradiometer. To measure the gradient magnetic field, two isotopically enriched ${ }^{87} \mathrm{Rb}$ vapor cells which are operated in the NMOR scheme are employed as the two sensors $\left(\mathrm{S}_{\mathrm{a}}\right.$ and $\left.\mathrm{S}_{\mathrm{b}}\right)$ of the gradiometer. The atomic spins of $\mathrm{S}_{\mathrm{a}}$ and $\mathrm{S}_{\mathrm{b}}$ are both synchronously stretched along $\hat{y}$ (polarized to the $\left.5 S_{1 / 2}, F=2, m_{F}=2\right\rangle$ state) by circularly polarized optical pumping and repumping with modulation frequency $\Omega_{m}[38,39]$. With the existence of a leading magnetic field $B_{L}$ along the $\hat{x}$-direction, the atomic spins of $\mathrm{S}_{\mathrm{a}}$ and $\mathrm{S}_{\mathrm{b}}$ start to undergo Larmor precession with Larmor frequencies of $\Omega_{L}^{a}$ and $\Omega_{L}^{b}$, respectively. The magnetic field gradient along the $x$ direction is given as $\partial B_{L} / \partial x=\left(\Omega_{L}^{a}-\Omega_{L}^{b}\right) /\left(\gamma D_{a b}\right)$, where $\gamma$ is the gyromagnetic ratio and $D_{a b}$ is the distance between the two sensors.

Entangled twin beams $\hat{a}_{1}$ and $\hat{b}_{1}$ are sent into $\mathrm{S}_{\mathrm{a}}$ and $\mathrm{S}_{\mathrm{b}}$ as the probe fields for the magnetic measurement. The frequencies of $a_{1}$ and $b_{1}$ are approximately $3 \mathrm{GHz}$ redand blue-detuned from the pump field for ${ }^{85} \mathrm{Rb}$ vapor, i.e. approximately $2 \mathrm{GHz}$ and $8 \mathrm{GHz}$ detuned from the transition $\left|5^{2} S_{1 / 2}, F=2\right\rangle \rightarrow\left|5^{2} P_{1 / 2}, F=2\right\rangle$ of the ${ }^{87} \mathrm{Rb}$ atom, respectively. The two probe beams are both far off-resonant with the ${ }^{87} \mathrm{Rb}$ atomic transition, leading to a quantum nondemolition atom-light interaction which can be described as:

$$
\hat{H}=\beta_{k} \hat{S}_{z}^{k} \hat{J}_{z}^{k} \quad(k=a, b),
$$

where $\beta_{k}$ is the coupling constant, $\hat{S}_{z}^{k}=1 /(2 i)\left(\hat{k}_{x}^{\dagger} \hat{k}_{y}-\right.$ $\left.\hat{k}_{y}^{\dagger} \hat{k}_{x}\right)$ is the Stokes parameter, and $\hat{J}_{z}^{k}$ is the macroscopic atomic spin along the $\hat{z}$-axis for sensor $\mathrm{S}_{\mathrm{k}}$. $\mathrm{k}=\mathrm{a}$ and $\mathrm{b}$ represent $S_{a}$ and $S_{b}$, respectively.

After the twin beams propagate through the atomic sensors, the polarization angles of the two beams are rotated by $\Delta \theta_{a} \propto \beta_{a} \hat{J}_{z}^{a}$ and $\Delta \theta_{b} \propto \beta_{b} \hat{J}_{z}^{b}$, which are related to the respective magnetic fields on the atomic sensors. The rotated output beams are designated $\hat{a}_{2}$ and $\hat{b}_{2}$. Each beam is split by a PBS, converting the rotation angles into intensity modulation. The final output fields $\hat{a}_{x}^{\text {out }}$ and $\hat{b}_{y}^{\text {out }}\left(\hat{a}_{y}^{\text {out }}\right.$ and $\left.\hat{b}_{x}^{\text {out }}\right)$ are mixed and detected by a balanced photodetector (BPD, Thorlabs PDB450A) with two photodiodes (S 3883) of high quantum efficiency $(96 \%)$ obtaining optical rotation signal $I$. The fluctuation of optical rotation signal $I$, which denotes the noise of the quantum magnetometer, is (see 

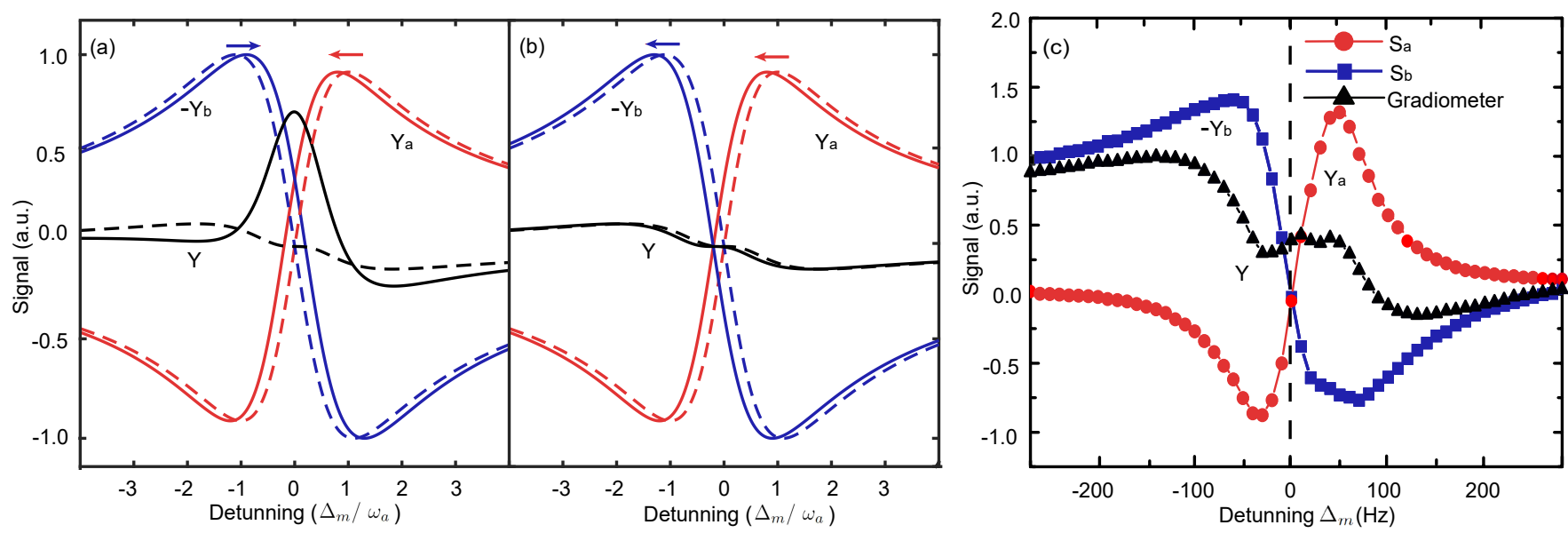

FIG. 3. (a,b) Schematic diagram of the magnetic resonances with a change of magnetic field gradient (a) and CMM field (b). The red and blue lines represent the magnetic resonances $\left[Y_{a}\right.$ and $\left.\left(-Y_{b}\right)\right]$ of the two sensors, while the black line denotes the subtracted magnetic resonance $(Y)$. When we apply a small magnetic field change, the magnetic resonances move from the dashed lines to the solid lines. (a) By applying a magnetic field gradient, the magnetic resonances (blue/red) move in the opposite direction. The subtracted magnetic resonance (black) changes obviously. (b) After applying a CMM field, both magnetic resonances (blue/red) move in the same direction. The change in the subtracted magnetic resonance (black) is inappreciable. (c) Measured magnetic resonances. The line with red circles and the line with blue squares represent the magnetic resonances of $\mathrm{S}_{\mathrm{a}}$ with linewidth $w_{a}=37 \mathrm{~Hz}$, and $\mathrm{S}_{\mathrm{b}}$ with linewidth $w_{b}=43 \mathrm{~Hz}$, which are measured by blocking optical pumping and repumping of $\mathrm{S}_{\mathrm{a}}$ and $\mathrm{S}_{\mathrm{b}}$, respectively. The line with the black triangles is obtained by "turning on" both sensors simultaneously. The dashed line denotes the frequency where we measure the sensitivity.

details in the Methods):

$$
\begin{aligned}
\operatorname{Var}(I) & =\left(G^{2}-g^{2} \cos \left(4 \theta_{0}\right)\right)\left|\alpha^{2}\right|+2 \sin ^{2}\left(2 \theta_{0}\right) g^{2}, \\
& =\operatorname{Var}_{\mathrm{PSN}}-2 g^{2}\left(\left|\alpha^{2}\right|-1\right) \cos ^{2}\left(2 \theta_{0}\right)
\end{aligned}
$$

where $\alpha$ is the amplitude of the two probe beams and $\theta_{0}$ is the relative angle between the optical axis of the half-wave plate and the $\hat{x}$ axis, $\operatorname{Var}_{\mathrm{PSN}}$ is the PSN with a power equal to the total power of the twin beams.

With the perturbations $\Delta \theta_{a}$ and $\Delta \theta_{b}$, the change in the optical rotation signal is (see details in the Methods)

$$
\begin{aligned}
\Delta I & =2 g^{2} \sin \left(2 \theta_{0}\right)|\alpha|^{2}\left(\Delta \theta_{b}-\Delta \theta_{a}\right) \\
& \propto \beta_{a} \cos \left(\Omega_{L}^{a} t\right)-\beta_{b} \cos \left(\Omega_{L}^{b} t\right),
\end{aligned}
$$

where $\Delta \theta_{b}-\Delta \theta_{a}$ contains the amplitude of the magnetic field gradient. To obtain the magnetic field gradient, we demodulate optical rotation signal $\Delta I$ with modulation frequency $\Omega_{m}$ and obtain the quadrature component of the magnetic resonance $Y$ [40],

$$
\begin{aligned}
Y & =Y_{a}-Y_{b} \\
& =\frac{A_{a} w_{a}\left(\Omega_{L}^{a}-\Omega_{m}\right)}{w_{a}^{2}+\left(\Omega_{L}^{a}-\Omega_{m}\right)^{2}}-\frac{A_{b} w_{b}\left(\Omega_{L}^{b}-\Omega_{m}\right)}{w_{b}^{2}+\left(\Omega_{L}^{b}-\Omega_{m}\right)^{2}},
\end{aligned}
$$

where $Y_{a}$ and $Y_{b}$ are the magnetic resonances from $\mathrm{S}_{\mathrm{a}}$ and $\mathrm{S}_{\mathrm{b}}$, respectively, with amplitude $A_{a, b} \propto \beta_{a, b}$ and linewidth $w_{a, b} \propto 1 / \tau_{a, b} . \quad \tau_{a}$ and $\tau_{b}$ are the coherence times for the two atomic sensors. To operate the gradiometer, we measure the magnetic resonance $Y$ by scanning the detuning $\Delta_{m}$ between $\Omega_{L}$ and $\Omega_{m}$ so as to find the optimal $\Omega_{m}$, where $Y$ is sensitive to the magnetic field gradient and insensitive to the CMM field. For $\Omega_{L}^{a}=\Omega_{L}^{b}=\Omega_{m}$, the response of gradiometer to the change of magnetic field is

$$
\begin{aligned}
\delta Y & =\frac{\partial Y_{a}}{\partial \Omega_{L}^{a}} \delta \Omega_{L}^{a}-\frac{\partial Y_{b}}{\partial \Omega_{L}^{b}} \delta \Omega_{L}^{b} \\
& =\left(k_{a}-k_{b}\right) \delta \Omega_{L}+\frac{k_{a}+k_{b}}{2} \delta \Omega_{L}^{g},
\end{aligned}
$$

where $k_{a, b}=\partial Y_{a, b} / \partial \Omega_{L}^{a, b}=A_{a, b} / w_{a, b}$ denote the slopes of the magnetic resonances from $\mathrm{S}_{\mathrm{a}}$ and $\mathrm{S}_{\mathrm{b}} . \quad \delta \Omega_{L}=$ $\left(\delta \Omega_{L}^{a}+\delta \Omega_{L}^{b}\right) / 2$ denotes the CMM noise, and $\delta \Omega_{L}^{g}=$ $\delta \Omega_{L}^{a}-\delta \Omega_{L}^{b}$ denotes the gradient field signal.

The magnetic resonances with $k_{a}=k_{b}$ are plotted in Figs. 3 (a) and (b) as a function of the detuning $\Delta_{m}=$ $\Omega_{L}-\Omega_{m}$ obtained by scanning $\Omega_{m}$. The dashed lines represent the magnetic resonances $Y$ (black dashed line), $Y_{a}$ (red dashed line) and $\left(-Y_{b}\right)$ (blue dashed line) without any magnetic field gradient, that is, $\Omega_{L}^{a}=\Omega_{L}^{b}$. As shown in Fig. 3 (a), by applying a magnetic field gradient $\delta \Omega_{L}^{g}$, that is, $\Omega_{L}^{a} \neq \Omega_{L}^{b}$, the two magnetic resonances $Y_{a}$ (red solid line) and $\left(-Y_{b}\right)$ (blue solid line) move in opposite directions, so that the magnetic resonance $Y$ (black solid line) changes dramatically at the $\Delta_{m}=0$, exhibiting that the most sensitive point for gradient field measurement is the zero-crossing point $\left(\Omega_{L}^{a}=\Omega_{L}^{b}=\Omega_{m}\right)$. On the other hand, as shown in Fig. 3 (b), by applying a small CMM field change $\delta \Omega_{L}$, both the magnetic resonances $Y_{a}$ and $\left(-Y_{b}\right)$ move in the identical direction, then the corresponding magnetic resonance $Y$ is shifted in the 

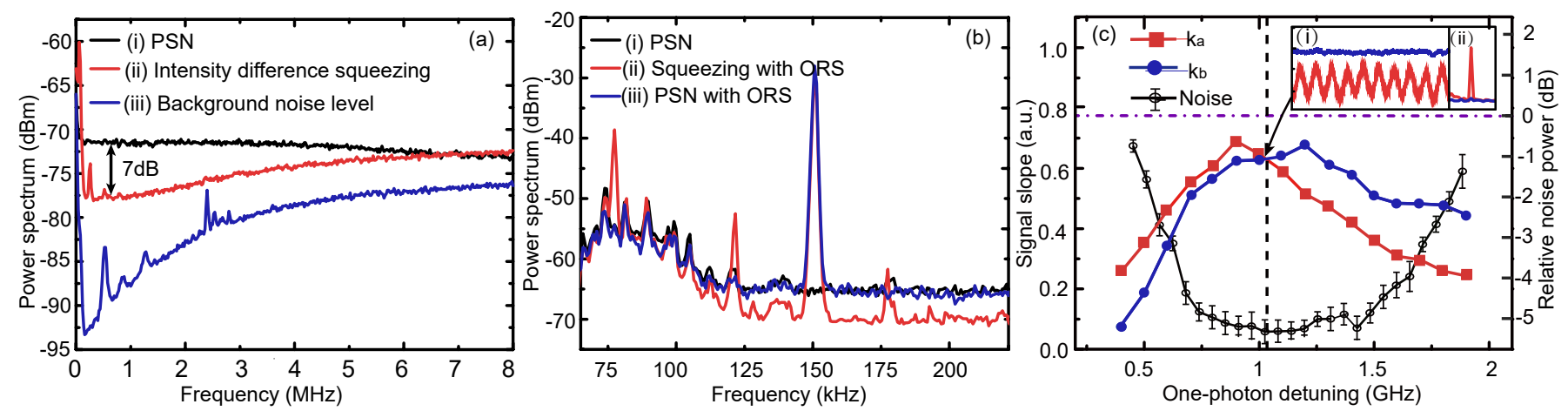

FIG. 4. (a) Noise power spectrum directly measured after the GT polarizer. The BPD's transimpedance gain is $10^{5} \mathrm{~V} / \mathrm{A}$ with a bandwidth of $5 \mathrm{MHz}$. The resolution bandwidth (RBW) and video bandwidth (VBW) are $30 \mathrm{kHz}$ and $300 \mathrm{~Hz}$, respectively. The peaks appearing at low frequency are electronic noises. (i) The black line denotes the PSN. (ii) The red line denotes the intensity-difference squeezing between the twin beams. (iii) The blue line denotes the background noise level. (b) Power spectrum measured after the ${ }^{87} \mathrm{Rb}$ cells. To measure the optical rotation signal at $152 \mathrm{kHz}$ and avoid electronic noise, the BPD transimpedance gain is $10^{6} \mathrm{~V} / \mathrm{A}$ with a bandwidth of $300 \mathrm{kHz}$. (i) The red line denotes the intensity-difference squeezing with an optical rotation signal at $152 \mathrm{kHz}$. (ii) The blue line denotes the PSN with an optical rotation signal at $152 \mathrm{kHz}$ from $\mathrm{S}_{\mathrm{a}}$. (iii) The black line denotes the PSN. RBW $=1 \mathrm{kHz}, \mathrm{VBW}=300 \mathrm{~Hz}$. (c) The slopes of the magnetic resonances of $\mathrm{S}_{\mathrm{a}}$ ( the line with red squares, left vertical scale) and $S_{b}$ (the line with solid blue circles, left vertical scale) and the relative noise power (the line with hollow black circles, right vertical scale) change with one-photon detuning. The horizontal dash-dotted line shows noise with PSN, and is taken as the reference $0 \mathrm{~dB}$ (right vertical scale). The vertical dashed line denotes the frequency where we finally measure the sensitivity. Inset: (i) the measured magnetic signal and (ii) their Fourier transform of $\mathrm{S}_{\mathrm{a}}$ (red line) and gradiometer (blue line). A CMM field oscillating at $5 \mathrm{~Hz}$ is effectively suppressed by gradiometer.

same direction. In this case, the magnetic resonance $Y$ does not response to the CMM field at $\Delta_{m}=0$. Thus, the zero-crossing point is also the best working point to immune CMM noise.

The experimental results of the magnetic resonances of gradiometer (the line with black triangles), sensor $\mathrm{S}_{\mathrm{a}}$ (the line with red circles) and $\mathrm{S}_{\mathrm{b}}$ (the line with blue squares) are given in Fig. 3 (c). Evidently, in the middle region around $\Delta_{m}=0$, the magnetic resonance $Y$ is insensitive to the CMM noise.

Noise reduction. The sensitive measurement of magnetic field at low frequencies has been a great challenge for practical applications [12-14]. To realize an ultra-sensitive quantum gradiometer at the frequencies below $\mathrm{kHz}$, the technologies developed above, including sensing magnetic field via NMOR, squeezing quantum noise with entangled light, and reducing CMM noise by gradiometic detection are integrated to achieve as large a signal as possible, remain the CMM noise minimized, and suppress quantum noise below the PSN.

Before entering atomic sensors, the intensity-difference noise of entangled twin beams $\hat{a}_{1}$ and $\hat{b}_{1}$ from FWM process is detected and given in Fig. 4 (a). Here, the squeezing spans a frequency range from $125 \mathrm{kHz}$ to $6 \mathrm{MHz}$ and the maximum squeezing is $7 \mathrm{~dB}$ at $0.6 \mathrm{MHz}$. To find the optimal $\Omega_{L}$ to perform magnetic measurement, one of the two sensors is "turned off" by blocking the optical pumping and repumping of this sensor, e.g., $\mathrm{S}_{\mathrm{b}}$. The power spectrum of the detected optical rotation signal oscillating at $\Omega_{L}^{a}$ is plotted in Fig. 4 (b). The intensity-difference squeezing of the twin beams is naturally mapped into the power spectrum. To determine the magnetic field from the detected optical rotation signal with quantum squeezing, one needs to demodulate this signal with a frequency of $\Omega_{m}=\Omega_{L}^{a}$. A particular $\Omega_{L}^{a}=152 \mathrm{kHz}$ is chosen here to optimize the sensitivity of magnetic measurements by balancing the different influences from nonlinear Zeeman (NLZ) effect, optical noise squeezing, and the residual magnetic resonance of the $F=1$ state (see details in the Methods). The available squeezing at $\Omega_{L}^{a}=152 \mathrm{kHz}$ remains $5.5 \mathrm{~dB}$ due to the absorption losses of the two ${ }^{87} \mathrm{Rb}$ cells $\left(11 \%\right.$ for $\mathrm{S}_{\mathrm{a}}$ and $7 \%$ for $\left.\mathrm{S}_{\mathrm{b}}\right)$.

To cooperate the reduction of CMM noise and suppression of quantum noise simultaneously, both sensors are "turned on" by sending optical pumping and repumping beams into them. Then, $\hat{a}_{2}$ and $\hat{b}_{2}$ are modulated with the Larmor precession of the atomic spin in $S_{a}$ and $S_{b}$, respectively. The optical rotation signal is demodulated and detected to give the difference between the magnetic resonances from $\mathrm{S}_{\mathrm{a}}$ and $\mathrm{S}_{\mathrm{b}}$. In this work, as the probes of gradiometer, the twin beams generated from an nondegenerate FWM process have different frequencies, leading to the different linewidths and amplitudes of the two magnetic resonances. However, the slopes of the magnetic resonances still can be set to the same, $k_{a}=k_{b}$, by tuning the one-photon detuning $\Delta$, since the frequencies of the twin beams change following the one-photon detuning. As seen in Fig. 4 (c), a crossover point, where $k_{a}=k_{b}$ is achieved at $\Delta=1.02 \mathrm{GHz}$. The common-mode rejection ratio (CMRR) of the gradiometer reaches 50 as shown in 
the inset of Fig. 4 (c), which is measured by applying a CMM field and detecting the residual of the CMM field in the difference. Meanwhile, quantum noise squeezing can still remain optimal in $5.5 \mathrm{~dB}$.

Sensitivity enhancement. After noise optimization in Fig. 4, the optimal point with $\Omega_{m}=\Omega_{L}=152 \mathrm{kHz}$ and $\Delta=1.02 \mathrm{GHz}$ are chosen to measure the magnetic field and gradient field sensitivities of the sensors which are given in Fig. 5. The sensitivity with a single sensor

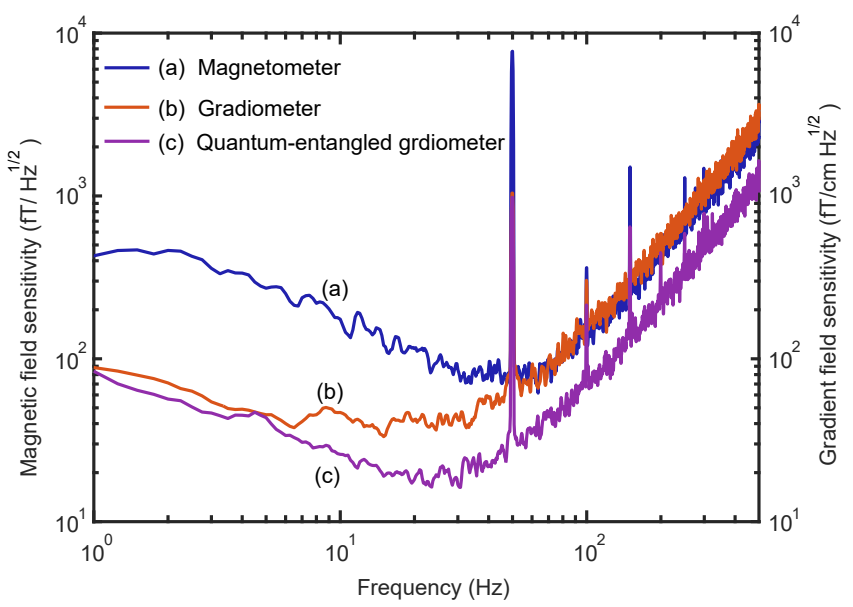

FIG. 5. Magnetic field and gradient field sensitivities. The transfer function of the low-pass filter is $b / \sqrt{f^{2}+b^{2}}$, where the bandwidth $b=\left(w_{a}+w_{b}\right) / 2=40 \mathrm{~Hz}$. (a) Sensitivity of the magnetometer limited by PSN and CMM noise. (b) Gradient field sensitivity of the gradiometer limited by PSN. (c) Gradient field sensitivity of the gradiometer with $5.5 \mathrm{~dB}$ squeezing.

$\mathrm{S}_{\mathrm{a}}$ is shown in the blue line (a) of Fig. 5, which is limited by both CMM noise and PSN. As a comparison, the red line (b) shows the sensitivity of the classical gradiometer using two coherent probe beams. Evidently, the CMM noise is suppressed in this gradiometer, and the noise floor is lowered to PSN limit $\sim 35 \mathrm{fT} / \mathrm{cm} \sqrt{\mathrm{Hz}}$. When the entangled twin beams replace the coherent beams as the probes of two sensors in quantum gradiometer, the sensitivity is further improved starting from $7 \mathrm{~Hz}$ with the help of $5.5 \mathrm{~dB}$ squeezing as shown in the purple line (c) of Fig. 5. The optimal sensitivity $18 \mathrm{fT} / \mathrm{cm} \sqrt{\mathrm{Hz}}$ is achieved at $20 \mathrm{~Hz}$. The sensitivity starts getting worse at both low and high frequencies due to the 1 /f noise and the bandwidth-limited frequency response of each sensor, respectively. The frequency response of each sensor, which acts as a low-pass filter with the transfer function $b / \sqrt{f^{2}+b^{2}}$, leads to the decrease of the signal at the increasing measurement frequency $f$ beyond the bandwidth $b$, and hence the reduction of the SNR.

\section{Conclusions and outlook.}

We construct and demonstrate a way to achieve simultaneous suppresses in both PSN and CMM noise by combining the techniques of quantum enhancement with gradiometic detection. With a $5.5 \mathrm{~dB}$ squeezed degree and a CMRR of 50, the sensitivity is enhanced from the sub-picotesla to 10-femtotesla level. The gradient field sensitivity reaches $18 \mathrm{fT} / \mathrm{cm} \sqrt{\mathrm{Hz}}$ with a $1 \mathrm{~cm}$ baseline at $20 \mathrm{~Hz}$, which is comparable to the sensitivity of the classical gradiometer operating with orders of magnitude more atoms. To the best of our knowledge, this is the first demonstration of quantum enhancement of the magnetic sensitivity at the low frequencies starting from $7 \mathrm{~Hz}$. Such quantum-enhanced magnetometry is promising for practical applications where the characteristic magnetic signatures range from $1 \mathrm{~Hz}$ to $1000 \mathrm{~Hz}$ in challenging environments [45].

The scheme of the quantum gradiometer is suitable for all magnetometry operated in NMOR type. There are still many possibilities to improve the performance of magnetometry with the technical routes of combining the quantum manipulation and atom-light interface. The bandwidth of magnetometry can be largely improved in several ways such as phase-locked loop [7] and selfoscillating [44] to achieve a better sensitivity at high frequency. The low frequency $1 / \mathrm{f}$ noise appears in Fig. 5 $(\mathrm{b}, \mathrm{c})$ caused by temperature shifting in the shield can be decreased by a better temperature control system. Moreover, in this work, the spin projection noise is not a limiting mechanism of the sensitivity since the used atom number in the sensors is relatively low. For optimized magnetometry with comparable noises from atom and light, the sensitivity can still be improved with the use of correlated twin beams (see details in the methods).

\section{Online content.}

Any methods, additional references, Nature Research reporting summaries, source data, extended data, supplementary information, acknowledgements, peer review information; details of author contributions and competing interests; and statements of data and code availability are available at XXX

\section{References.}

[1] Budker, D., Gawlik, W., Kimball, D, F. et al. Resonant nonlinear magneto-optical effects in atoms. Rev. Mod. Phys. 74, 1153 (2002).

[2] Kominis, I, K., Kornack, T, W., Allred, J, C. et al. A subfemtotesla multichannel atomic magnetometer. Nature. 422, 596 (2003).
[3] Budker, D., Romalis, M. Optical magnetometry. Nat. Phys. 3, 227 (2007).

[4] Ledbetter, M, P., Savukov, I, M., Acosta, V, M. et al. Spin-exchange-relaxation-free magnetometry with Cs vapo. Phys. Rev. A. 77, 033408 (2008).

[5] Dang, H, B., Maloof, A, C., Romalis, M, V. Ultrahigh 
sensitivity magnetic field and magnetization measurements with an atomic magnetometer. Appl. Phys. Lett. 97, 151110 (2010).

[6] Sheng, D., Li, S., Dural, N. et al. Subfemtotesla scalar atomic magnetometry using multipass cells. Phys. Rev. Lett. 110, 160802 (2013).

[7] Sheng, D., Perry, A, R., Krzyzewski, S, P. et al. A microfabricated optically-pumped magnetic gradiometer. Appl. Phys. Lett. 110, 031106 (2017).

[8] Vasilakis, G., Brown, J, M., Kornack, T, W. et al. Limits on New Long Range Nuclear Spin-Dependent Forces Set with a K- He 3 Comagnetometer . Phys. Rev. Lett. 103, 261801 (2009).

[9] Altarev, I., Baker, C, A., Ban, G. et al. Test of Lorentz invariance with spin precession of ultracold neutrons. Phys. Rev. Lett. 103, 081602 (2009).

[10] Jiang, M., Su, H., Garcon, A., Peng, X., Budker, D.. Search for axion-like dark matter with spin-based amplifiers. arXiv preprint arXiv:2102.01448 (2021).

[11] Safronova, M. S., Budker, D., DeMille, D., Kimball, D. F. J., Derevianko, A., Clark, C. W. Search for new physics with atoms and molecules. Rev. Mod. Phys. 90, 025008 (2018).

[12] Boto, E., Holmes, N., Leggett, J. et al. Moving magnetoencephalography towards real-world applications with a wearable system. Nature. 555 (7698): 657-661, 657 (2018).

[13] Hill, R, M., Boto, E., Holmes, N. et al. A tool for functional brain imaging with lifespan compliance. Nat. Commun. 10, 4785 (2019).

[14] Jensen, K., Skarsfeldt, M, A., Stærkind, H. et al. Magnetocardiography on an isolated animal heart with a roomtemperature optically pumped magnetomete. Sci. Rep. 8, 1 (2019).

[15] Kim, Y, J., Savukov, I., Huang, J, H. et al. Magnetic microscopic imaging with an optically pumped magnetometer and flux guides. Appl. Phys. Lett. 110, 043702 (2017).

[16] Chatzidrosos, G., Wickenbrock, A., Bougas, L. et al. Eddy-current imaging with nitrogen-vacancy centers in diamond. Phys. Rev. Applied 11, 014060 (2019).

[17] Hu, Y., Iwata, G, Z., Mohammadi, M. et al. Sensitive magnetometry reveals inhomogeneities in charge storage and weak transient internal currents in Li-ion cells. PNAS 117 (20), 10667-10672 (2020).

[18] Bustos, F, P., Calia, D, B., Budker, D. et al. Remote sensing of geomagnetic fields and atomic collisions in the mesosphere. Nat. Commun. 9 (1): 1-8, 3981 (2018).

[19] Auzinsh, M., Budker, D., Kimball, D, F. et al. Can a quantum nondemolition measurement improve the sensitivity of an atomic magnetometer? Phys. Rev. Lett. 93 (17), 173002 (2004).

[20] Wolfgramm, F., Cere, A., Beduini, F, A. et al. Squeezedlight optical magnetometry. Phys. Rev. Lett. 105 (5), 053601 (2010).

[21] Horrom, T., Singh, R., Dowling, J, P. et al. Quantumenhanced magnetometer with low-frequency squeezing. Phys. Rev. A. 86 (2), 023803 (2012).

[22] Otterstrom, N., Pooser, R, C., Lawrie, B, J. Nonlinear optical magnetometry with accessible in situ optical squeezing. Opt. Lett. 39 (22), 6533-6536 (2014).

[23] Bao, G., Wu, S., Liu, S. et al. Enhancement of the signalto-noise ratio of an atomic magnetometer by $10 \mathrm{~dB}$. Phys. Rev. Applied. 11 (5), 054075 (2019).
[24] Bai, L., Wen, X., Yang, Y., Zhang, L. et al. Quantumenhanced rubidium atomic magnetometer based on Faraday rotation via $795 \mathrm{~nm}$ stokes operator squeezed light. J. Opt. 23 (8), 085202 (2021).

[25] Troullinou, C., Jiménez-Martínez, R., Kong, J. et al. Squeezed-Light Enhancement and Backaction Evasion in a High Sensitivity Optically Pumped Magnetometer. Phys. Rev. Lett. 127(19), 193601 (2021).

[26] Wasilewski, W., Jensen, K., Krauter, H. et al. Quantum noise limited and entanglement-assisted magnetometry. Phys. Rev. Lett. 104 (13), 133601 (2010).

[27] Bao, H., Duan, J., Jin, S. et al. Spin squeezing of 10 11 atoms by prediction and retrodiction measurements. Nature. 581 (7807), 159-163 (2020).

[28] Shah, V, K., Wakai, R, T. A compact, high performance atomic magnetometer for biomedical applications. Phys. Med. Biol. 58 (22), 8153 (2013).

[29] Kamada, K., Ito, Y, Ichihara, S. et al. Noise reduction and signal-to-noise ratio improvement of atomic magnetometers with optical gradiometer configurations. Opt. Express. 23, 6976 (2015).

[30] Jiang, M., Frutos, R, P., Wu, T. et al. Magnetic gradiometer for the detection of zero-to ultralow-field nuclear magnetic resonance. Phys. Rev. Applied. 11, 024005 (2019).

[31] Lucivero, V, G., Lee, W., Dural, N. et al. Femtotesla direct magnetic gradiometer using a single multipass cell. Phys. Rev. Applied 15, 014004 (2021).

[32] Boyer, V., Marino, A, M., Pooser, R, C. et al. Entangled images from four-wave mixing. Science. 321, 544547 (2008).

[33] McCormick, C, F., Boyer, V., Arimondo, E. et al. Strong relative intensity squeezing by four-wave mixing in rubidium vapor. Opt. Lett. 32, 178-180 (2007).

[34] Hudelist, F., Kong, J., Liu, C. et al. Quantum metrology with parametric amplifier-based photon correlation interferometers. Nat. Commun. 5, 1-6 (2014).

[35] Qin, Z., Cao, L., Wang, H. et al. Experimental generation of multiple quantum correlated beams from hot rubidium vapor. Phys. Rev. Lett. 113, 023602 (2014).

[36] Gulati, G, K., Srivathsan, B., Chng, B. et al. Polarization entanglement and quantum beats of photon pairs from four-wave mixing in a cold $87 \mathrm{Rb}$ ensemble. New J. Phys. 17093034 (2015).

[37] Balabas, M, V., Karaulanov, T., Ledbetter, M, P. et al. Polarized alkali-metal vapor with minute-long transverse spin-relaxation time. Phys. Rev. Lett. 105, 070801 (2010).

[38] Han, R., Balabas, M., Hovde, C. et al. Is light narrowing possible with dense-vapor paraffin coated cells for atomic magnetometers? AIP Advances. 7, 125224 (2017).

[39] Jiang, Z, Y., Liu, X, C., Lin, P, W. et al. Enhanced fully optically pumped magnetic resonance with optical sideband auxiliary pumping. Opt. Lett. 45, 105-108 (2020).

[40] Auzinsh, M., Budker, D., Rochester, S. Optically polarized atoms: understanding light-atom interactions (Oxford University, New York, 2010).

[41] Bao, G., Wickenbrock, A., Rochester, S. et al. Suppression of the nonlinear Zeeman effect and heading error in Earth-field-range alkali-vapor magnetometers. Phys. Rev. Lett. 120.033202 (2018).

[42] Wang, Z., Peng, X., Zhang, R. et al. Single-Species Atomic Comagnetometer Based on Rb 87 Atoms. Phys. Rev. Lett. 124, 193002 (2020). 
[43] Lucivero, V, G., Anielski, P., Gawlik, W. et al. Shotnoise-limited magnetometer with sub-picotesla sensitivity at room temperature. Rev. Sci. Instrum. 85, 113108 (2014).

[44] Higbie, J, M., Corsini, E., Budker, D., Robust. highspeed, all-optical atomic magnetometer. Rev. Sci. Instrum. 77, 113106 (2006).

[45] Fu, K, M, C., Iwata, G, Z., Wickenbrock, A. et al. Sensitive magnetometry in challenging environments. AVS Quantum Sci. 2, 044702 (2020).

\section{Data availability.}

Data that support the plots within this paper and other findings of this study are available from the corresponding authors upon reasonable request. Source data are provided with this paper.

\section{Acknowledgements.}

We would like to thank D. Budker from Johannes Gutenberg University for useful discussions. We acknowledge financial support from the National Key Research and Development Program of China (Grant No. 2016YFA0302001), the National Natural Science Foundation of China (Grant Nos. 11654005, 11874152, 91536114, and 11904227), the Shanghai Municipal Science and Technology Major Project (Grant No. 2019SHZDZX01), the Fellowship of China Postdoctoral Science Foundation (Grant No. 2020TQ0193, 2021M702146, 2021M702150), the Sailing Program of the Science and Technology Commission of Shanghai Municipality (19YF1421800), Innovation Program of Shanghai Municipal Education Commission No. 202101070008E00099, and the Fundamental Research Funds for the Central Universities. W.Z. acknowledges additional support from the Shanghai Talent Program.

\section{Methods.}

Detail for the theory We generate intensity correlated two-mode squeezed fields by PA process in hot ${ }^{85} \mathrm{Rb}$ vapor. By mixing and amplifying the two field modes $\hat{a}_{i n}$ and $\hat{b}_{i n}$, correlations between two modes are generated.

$$
\left[\begin{array}{l}
\hat{a}_{x} \\
\hat{a}_{x}^{\dagger} \\
\hat{b}_{x} \\
\hat{b}_{x}^{\dagger}
\end{array}\right]=\left[\begin{array}{cccc}
G & 0 & 0 & g \\
0 & G & g & 0 \\
0 & g & G & 0 \\
g & 0 & 0 & G
\end{array}\right]\left[\begin{array}{c}
\hat{a}_{i n} \\
\hat{a}_{i n}^{\dagger} \\
\hat{b}_{i n} \\
\hat{b}_{i n}^{\dagger}
\end{array}\right]
$$

where, $G^{2}-g^{2}=1$. In our system, with a coherent state $|\alpha\rangle$ input at port $\hat{a}_{i n}$ and a vacuum state at port $\hat{b}_{i n}, \hat{a}_{x}$ and $\hat{b}_{x}$ are the outputs of the PA process. Both of the fields are linearly polarized along $x$ direction.

$\hat{a}_{x}$ and $\hat{b}_{x}$ act as the probe beams of two ${ }^{87} \mathrm{Rb}$ vapor sensors $\hat{a}_{1}$ and $\hat{b}_{1}$ and couple the magnetic field into their polarizations. The polarizations of $\hat{a}_{2}$ and $\hat{b}_{2}$ are rotated with angles $\Delta \theta_{a}$ and $\Delta \theta_{b} . \hat{a}_{2}$ and $\hat{b}_{2}$ are further rotated by a HWP with angle $\theta_{0}$ and mixed with a vacuum state with $y$ polarization $\hat{a}_{y}^{i n}$ and $\hat{b}_{y}^{i n}$ at the PBS, respectively. The output of the PBS is:

$$
\left[\begin{array}{l}
\hat{a}_{x}^{\text {out }} \\
\hat{a}_{y}^{\text {out }} \\
\hat{b}_{x}^{\text {out }} \\
\hat{b}_{y}^{\text {out }}
\end{array}\right]=\left[\begin{array}{cccc}
\cos \left(\theta_{a}\right) & -\sin \left(\theta_{a}\right) & 0 & 0 \\
\sin \left(\theta_{a}\right) & \cos \left(\theta_{a}\right) & 0 & 0 \\
0 & 0 & \cos \left(\theta_{b}\right) & -\sin \left(\theta_{b}\right) \\
0 & 0 & \sin \left(\theta_{b}\right) & \cos \left(\theta_{b}\right)
\end{array}\right]\left[\begin{array}{c}
\hat{a}_{x} \\
\hat{a}_{y}^{\text {in }} \\
\hat{b}_{x} \\
\hat{b}_{y}^{\text {in }}
\end{array}\right]
$$

where $\theta_{a}=\theta_{0}+\Delta \theta_{a}, \theta_{b}=\theta_{0}+\Delta \theta_{b}$. The input-output relation of optical field after the whole process is:

$$
\left[\begin{array}{c}
\hat{a}_{x}^{\text {out }} \\
\hat{a}_{x}^{\text {out }} \\
\hat{a}_{y}^{\text {out }} \\
\hat{a}_{y}^{\text {out }} \\
\hat{b}_{x}^{\text {out }} \\
\hat{b}_{x}^{\text {out }} \\
\hat{b}_{y}^{\text {out }} \\
\hat{b}_{y}^{\text {out }}
\end{array}\right]=\left[\begin{array}{cccccccc}
G \cos \left(\theta_{a}\right) & 0 & 0 & g \cos \left(\theta_{a}\right) & -\sin \left(\theta_{a}\right) & 0 & 0 & 0 \\
0 & G \cos \left(\theta_{a}\right) & g \cos \left(\theta_{a}\right) & 0 & 0 & -\sin \left(\theta_{a}\right) & 0 & 0 \\
G \sin \left(\theta_{a}\right) & 0 & 0 & g \sin \left(\theta_{a}\right) & \cos \left(\theta_{a}\right) & 0 & 0 & 0 \\
0 & G \sin \left(\theta_{a}\right) & g \sin \left(\theta_{a}\right) & 0 & 0 & \cos \left(\theta_{a}\right) & 0 & 0 \\
0 & g \cos \left(\theta_{b}\right) & G \cos \left(\theta_{b}\right) & 0 & 0 & 0 & -\sin \left(\theta_{b}\right) & 0 \\
g \cos \left(\theta_{b}\right) & 0 & 0 & G \cos \left(\theta_{b}\right) & 0 & 0 & 0 & -\sin \left(\theta_{b}\right) \\
0 & g \sin \left(\theta_{b}\right) & G \sin \left(\theta_{b}\right) & 0 & 0 & 0 & \cos \left(\theta_{b}\right) & 0 \\
g \sin \left(\theta_{b}\right) & 0 & 0 & G \sin \left(\theta_{b}\right) & 0 & 0 & 0 & \cos \left(\theta_{b}\right)
\end{array}\right]\left[\begin{array}{c}
\hat{a}_{i n} \\
\hat{a}_{\text {in }}^{\dagger} \\
\hat{b}_{i n} \\
\hat{b}_{i n}^{\dagger} \\
\hat{a}_{y}^{\text {in }} \\
\hat{a}_{y}^{\text {in }} \\
\hat{b}_{y}^{\text {in }} \\
\hat{b}_{y}^{\text {in }}
\end{array}\right] .
$$

The output fields of PBS are sent to the balanced photodetector and achieve the intensity-difference measurement. The observation of magnetometer is:

$$
\hat{I}=\hat{I}_{x}^{a}-\hat{I}_{y}^{a}-\hat{I}_{x}^{b}+\hat{I}_{y}^{b}
$$

where, $\hat{I}_{x}^{a}=\hat{a}_{x}^{\text {out }}{ }^{\dagger} \hat{a}_{x}^{\text {out }}, \hat{I}_{y}^{a}=\hat{a}_{y}^{\text {out }} \hat{a}_{y}^{\text {out }}, \hat{I}_{x}^{b}=\hat{b}_{x}^{\text {out }} \hat{b}_{x}^{\text {out }}$,
$\hat{I}_{y}^{b}=\hat{b}_{y}^{\text {out }} \hat{b}_{y}^{\text {out }}$. The average value of $\hat{I}$ is:

$\langle\hat{I}\rangle=\left[G^{2} \cos \left(2 \theta_{a}\right)-g^{2} \cos \left(2 \theta_{b}\right)\right]\left|\alpha^{2}\right|+g^{2}\left[\cos \left(2 \theta_{a}\right)-\cos \left(2 \theta_{b}\right)\right]$.

When operate at $\theta_{a}=\theta_{b}=0$, the fluctuation of magnetometer is:

$$
\operatorname{Var}(\hat{I})=\left(1+g^{2}-g^{2} \cos \left(4 \theta_{0}\right)\right)\left|\alpha^{2}\right|+2 \sin ^{2}\left(2 \theta_{0}\right) g^{2}
$$

The change in the observation of magnetometer with per- 
turbation $\Delta \theta_{a}$ and $\Delta \theta_{b}$ is:

$$
\begin{aligned}
\Delta I & =\frac{\partial\langle\hat{I}\rangle}{\partial \theta_{1}} \Delta \theta_{a}+\frac{\partial\langle\hat{I}\rangle}{\partial \theta_{2}} \Delta \theta_{b} \\
& =2\left[g^{2} \sin \left(2 \theta_{2}\right) \Delta \theta_{b}-G^{2} \sin \left(2 \theta_{1}\right) \Delta \theta_{a}\right]\left|\alpha^{2}\right| \\
& +2 g^{2}\left[\sin \left(2 \theta_{2}\right) \Delta \theta_{b}-\sin \left(2 \theta_{1}\right) \Delta \theta_{a}\right] .
\end{aligned}
$$

For $\Delta \theta_{a} \ll \theta_{a}, \Delta \theta_{b} \ll \theta_{b}$ and $G \approx g \gg 1$, we have

$$
\Delta I=2 g^{2} \sin \left(2 \theta_{0}\right)\left|\alpha^{2}\right|\left(\Delta \theta_{b}-\Delta \theta_{a}\right) .
$$

Hence the signal-to-noise ratio (SNR) is:

$$
\frac{(\Delta I)^{2}}{\operatorname{Var}(\hat{I})}=\frac{4 g^{4} \sin ^{2}\left(2 \theta_{0}\right)\left|\alpha^{4}\right|\left(\Delta \theta_{b}-\Delta \theta_{a}\right)^{2}}{\left(1+g^{2}-g^{2} \cos \left(4 \theta_{0}\right)\right)\left|\alpha^{2}\right|+2 \sin ^{2}\left(2 \theta_{0}\right) g^{2}}
$$

The SNRs of the gradiometer change with $g$ are plot-

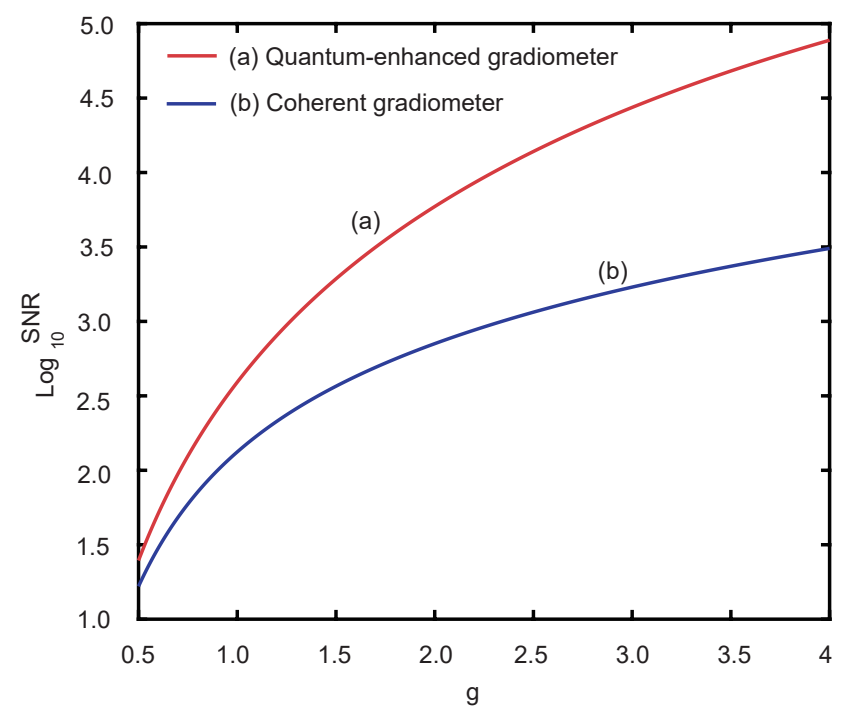

FIG. 6. The SNR of quantum-enhanced gradiometer (a) and coherent gradiometer (b) change with $g$. Here, we set $\alpha=100$, $\theta_{0}=0.05$ and $\left(\Delta \theta_{b}-\Delta \theta_{a}\right)^{2}=1$.

ted in Fig.6. The red line and the blue line represent the SNR of quantum-enhanced gradiometer and coherent gradiometer, respectively. We can see that with the increase of $g$, the SNR of the squeezed gradiometer is always better than the coherent gradiometer.

Fundamental limitation of sensitivity. For magnetometry based on optical readout of atomic ensemble's spin precession, the sensitivity is fundamentally limited PSN and SPN. The corresponding sensitivity $\delta B_{\mathrm{ph}}$ and $\delta B_{\text {at }}$ can be described as [19]:

$$
\begin{aligned}
& \delta B_{\mathrm{ph}}=\frac{1}{\gamma \tau} \frac{1}{N_{\mathrm{at}} \sqrt{N_{\mathrm{ph}}}} \frac{\Delta_{p}}{\Gamma_{0}} \frac{A}{\lambda^{2}}, \\
& \delta B_{\mathrm{at}}=\frac{1}{\gamma \tau} \frac{\sqrt{N_{\mathrm{ph}}}}{\sqrt{N_{\mathrm{at}}}} \frac{\Gamma_{0}}{\Delta_{p}}\left(\frac{\lambda^{2}}{A}\right)^{1 / 2},
\end{aligned}
$$

where $\gamma$ is the gyromagnetic ratio, $\tau$ is the measurement time. $N_{\text {at }}$ and $N_{\text {ph }}$ are the total number of atoms and photons used in the measurement, respectively. $\Gamma_{0}$ is the natural transition width, $\Delta_{p}$ is the frequency detuning from optical resonance and $\lambda$ is the light wavelength. $A$ is the cross section of the probe and assumed to match the atomic sample. Considering the noise from both atom and light, the sensitivity is limited by the larger one of $\delta B_{\text {at }}$ and $\delta B_{\mathrm{ph}}$. When the atom number and photon number satisfying $\sqrt{N_{\mathrm{at}}} N_{\mathrm{ph}}<\left(\Delta_{p} / \Gamma_{0}\right)^{2}\left(A / \lambda^{2}\right)^{1 / 2}$, we get $\delta B_{\mathrm{ph}}>\delta B_{\text {at }}$. The sensitivity is limited by PSN. In our work, $N_{\text {at }}$ is limited by the operation temperature of vapor cell and $N_{\mathrm{ph}}$ is limited by the FWM process. In consequence, the sensitivity of gradiometer with coherent light is fundamentally limited by PSN. With the use of a squeezed light, the sensitivity can further break PSN.

The overall sensitivity limitations with the change of $N_{\text {ph }}$ are plotted in Fig. 7. We can find the optimum number of photons and corresponding sensitivity by minimizing the overall uncertainty. In Fig. 7 (a), the sensitivity, which limited by photon noise with $5.5 \mathrm{~dB}$ squeezing and SPN, can reach optimal sensitivity $\delta B_{o}^{s}$ with optimal photon number $N_{\mathrm{oph}}^{s}$. In Fig. 7 (b), the sensitivity, which limited by PSN and SPN, can reach optimal sensitivity $\delta B_{o}^{c}$ with optimal photon number $N_{\mathrm{oph}}^{c}$. Compared with coherent light, the use of squeezed light can lead to a better sensitivity $\left(\delta B_{o}^{s}<\delta B_{o}^{c}\right)$ with lower number of photons $\left(N_{\mathrm{oph}}^{s}<N_{\mathrm{oph}}^{c}\right)$.

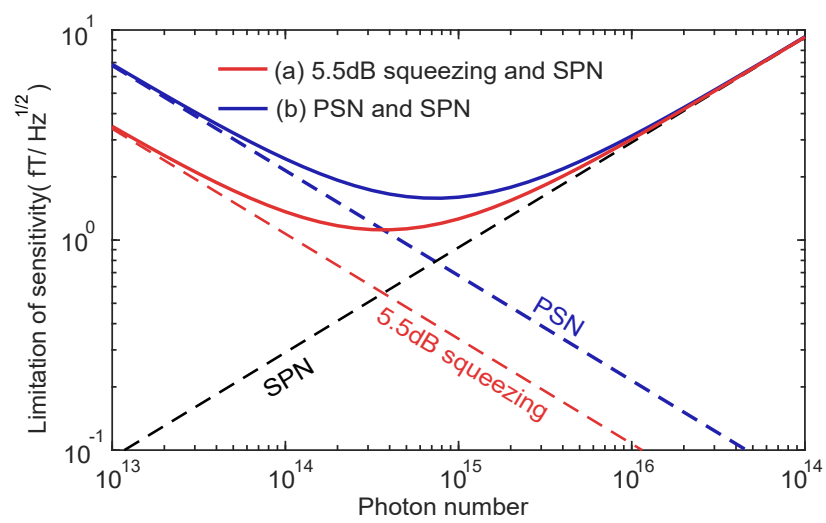

FIG. 7. (a) The sensitivity limited by photon noise with 5.5 $\mathrm{dB}$ squeezing and SPN. (b) The sensitivity limited by PSN and SPN. Here we choose $\gamma=7 \mathrm{~Hz} / \mathrm{nT}, \tau=1 \mathrm{~s}, \Delta_{p}=8 \mathrm{GHz}$, $\Gamma_{0}=5.7 \mathrm{MHz}, A=6 \times 10^{-4} \mathrm{~m}^{2}, \lambda=795 \mathrm{~nm}, N_{\text {at }}=10^{10}$ which are consistent with the experimental condition.

In ref. [19], the sensitivity is discussed in free induction decay scheme. When the number of spins that flip due to ground-state relaxation becomes comparable to unity, uncertainty due to relaxation begins to dominate the atomic noise. The sensitivity have no improvement with squeezed light. While in our work, the decayed spin are repolarized by synchronized pumping. With long measurement time, the sensitivity still can be improved by the squeezed light. 
Experimental layout. The cell generating FWM process contains isotopically pure ${ }^{85} \mathrm{Rb}$ vapor cell $(12.5 \mathrm{~mm}$ long) that can be heated to over $120^{\circ} \mathrm{C}$. It is antireflection (AR) coated on all four surfaces to achieve a high transmission efficiency $(\sim 95 \%)$. The seed $\hat{a}$ is red detuned approximately $3 \mathrm{GHz}$ from the pump beam by using an acousto-optic modulator (AOM) in double-pass configuration. Both FWM pump and seed are focused in the center of the cell with beam waists $500 \mu \mathrm{m}$ and $250 \mu \mathrm{m}$, respectively. In order to calibrate the PSN, the temperature of the ${ }^{85} \mathrm{Rb}$ vapor cell is set to $100^{\circ} \mathrm{C}$, there was no entanglement between the twin beams and the intensity of the twin beams is equated by the attenuator. Therefore, the noise level after intensity difference detection can be regarded as PSN.

The sensors $\left(\mathrm{S}_{\mathrm{a}}\right.$ and $\left.\mathrm{S}_{\mathrm{b}}\right)$ of the gradiometer are mainly constructed by two paraffin-coated and AR-coated cylindrical cells [37] $(1 \mathrm{~cm}$ in length; $1 \mathrm{~cm}$ in diameter $)$ which are placed side by side in a three-layer $\mu$-metal magnetic shield. The two cells are heated to $35^{\circ} \mathrm{C}$ for sensitivity optimization. To generate CMM field and magnetic field gradient on two sensors we add a set of Helmholtz coil and a set of anti-Helmholtz coil with a diameter of 92 $\mathrm{mm}$ outside of two ${ }^{87} \mathrm{Rb}$ vapor cells. For each sensor, the atoms are fully polarized to $5 S_{1 / 2}, F=2, m_{F}=2$ state by two circularly polarized, $\hat{y}$-directed laser beams $\left(\mathrm{P}_{2}^{\mathrm{a}} / \mathrm{P}_{3}^{\mathrm{a}}\right.$ and $\left.\mathrm{P}_{2}^{\mathrm{b}} / \mathrm{P}_{3}^{\mathrm{b}}\right)[38,39]$, where $\mathrm{P}_{2}^{\mathrm{a}}$ and $\mathrm{P}_{3}^{\mathrm{a}}$ are optical pumping and repumping for sensor $\mathrm{S}_{\mathrm{a}}$; $\mathrm{P}_{2}^{\mathrm{b}}$ and $\mathrm{P}_{3}^{\mathrm{b}}$ are optical pumping and repumping for sensor $\mathrm{S}_{\mathrm{b}}$. The optical pumping beam is resonant with $5 S_{1 / 2}, F=2$ to $5 P_{1 / 2}, F^{\prime}=2$ transition to polarize the atoms. The optical repumping beam is resonant with $5 S_{1 / 2}, F=1$ to $5 P_{1 / 2}, F^{\prime}=2$ transition to pump all the atoms to $F=2$ states. Both optical pumping and repumping beams are split and sent to the two sensors $\left(\mathrm{S}_{\mathrm{a}}, \mathrm{S}_{\mathrm{b}}\right)$ of the gradiometer. By synchronously modulating the intensity of optical pumping and repumping beams with a square-wave modulation (3\% duty cycle) by acousto-optic modulators (AOM), the atomic spins are periodically driven and start Larmor procession. The power of optical pumping and repumping beams during the "on" part of the cycle is $5 \mathrm{~mW}$ and $30 \mathrm{~mW}$, respectively. After the twin beams propagate through the atomic medium, the electric field amplitude $\varepsilon$, overall phase $\phi$, polarization angle $\theta$, and ellipticity $\epsilon$ are both modulated at Larmor frequency $\Omega_{L}[40]$.

Choice of Larmor frequency. To minimize the optical field noise, $\Omega_{m}$ should be chosen as the frequency $f_{s q}$ of the maximum squeezed degree, which means that the leading magnetic field $B_{L}$ should be chosen to satisfy $\Omega_{m}=\Omega_{L}=f_{s q}=0.6 \mathrm{MHz}$, where $B_{L}$ at the Gauss level is applied on the atomic sensor. This $B_{L}$ field is large enough to bring about the NLZ effect, inducing splitting and asymmetry of the magnetic resonance [41]. Moreover, with low $\Omega_{L}$, the magnetic resonance generated from the residual atoms in the $F=1$ state disturbs the main magnetic resonance from the $F=2$ state atoms due to the tiny difference in the gyromagnetic ratios of the $F=1$ and $F=2$ states [42]. The frequency difference between these two magnetic resonances increases with $B_{L}$. Consequently, we choose $\Omega_{L}$ to be $152 \mathrm{kHz}$ to balance the influences of the NLZ effect, noise squeezing and the interference of the magnetic resonance from $F=1$ state.

CMRR. In general, we use the CMRR to evaluate the

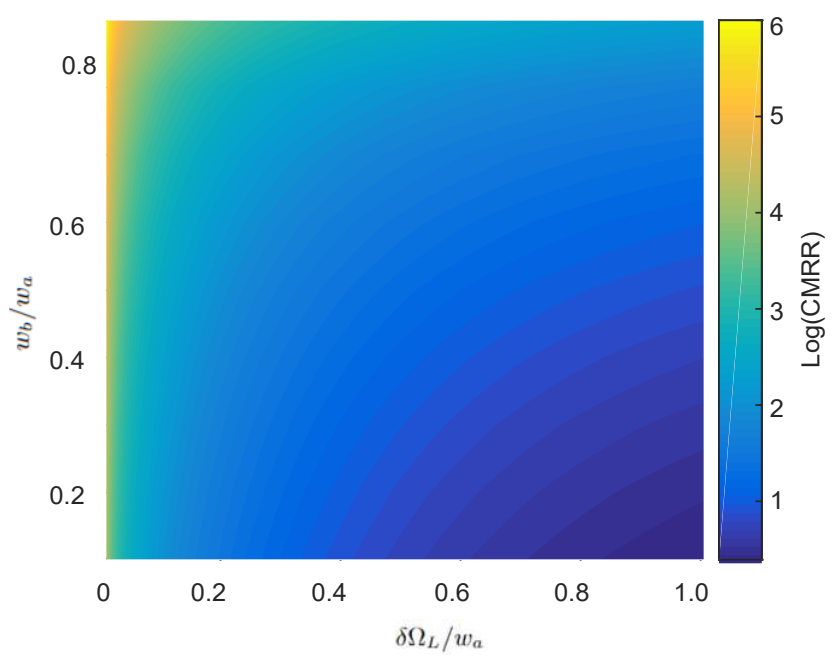

FIG. 8. CMRR change with $\delta \Omega_{L} / w_{a}$ and $w_{b} / w_{a}$.

reduction of CMM noise. When the two resonances have same amplitude and linewidth, the magnetic noise will be reduced completely. In practical, due to the limitation and influence of various conditions, there will be a difference between the linewidth of two sensors. To ensure two sensors have same response to the magnetic field, the gradiometer is operated with $k_{a}=k_{b}$. In this condition, the CMRR is related with ratio between the linewidth of magnetic resonances $w_{b} / w_{a}$ and the change of the CMM field $\delta \Omega_{L}$. The CMRR which is defined as the ratio of CMM field and the remaining portion of the CMM field in the difference [30] can be written as:

$$
\mathrm{CMRR}=\frac{w_{a}^{2}\left(w_{b}^{2}+\delta \Omega_{L}^{2}\right)}{\left(w_{a}^{2}-w_{b}^{2}\right) \delta \Omega_{L}^{2}} .
$$

The CMRR decrease with the increase of both $\delta \Omega_{L}$ and the difference between $w_{a}$ and $w_{b}$, as shown in Fig. 8 .

Author contributions. G.Z.B., and W.Z. conceived the research. G.Z.B, L.Q.C., and W.Z. designed the experiments. G.Z.B., S.H.W., J.X.G., J.C., W.D., P.Y.Y., and L.Q.C. performed the experiment. G.Z.B., S.H.W., W.D., and W.Z. contributed to the theoretical study. G.Z.B., S.H.W., analyzed the data. G.Z.B., S.H.W., and J.C. draw the diagrams. G.Z.B., S.H.W., L.Q.C., and W.Z. wrote the paper. All authors contributed to the discussion and review of the manuscript. W.Z. supervised the whole project.

Competing interests. The authors declare no competing interests. 
Correspondence and requests for materials should be addressed to Guzhi Bao. 